\title{
Un nouveau genre et une nouvelle espèce de l'ordre Stemmiulida du Viet-Nam (Diplopoda)
}

\author{
A new genus and species of the order Stemmiulida from Vietnam \\ (Diplopoda)
}

\section{Новые род и вид из отряда Stemmiulida из Вьетнама (Diplopoda)}

\author{
J.-P. Mauriès ${ }^{1}$, S. I. Golovatch ${ }^{2}$ \& J.-J. Geoffroy ${ }^{3}$ \\ ЖК.-П. Морьес ${ }^{1}$, С.И. Головач ${ }^{2}$, Ж.-ЖК. ЖКоффруа ${ }^{3}$
}

\footnotetext{
${ }^{1}$ Muséum national d'Histoire naturelle, Département Systématique et Evolution, UMR7205, Case postale 53, 61, rue Buffon, F-75231 Paris, France.

${ }^{2}$ Институт проблем экологии и эволюции РАН, Ленинский пр., 33, Москва 119071 Россия.

${ }^{2}$ Institute for Problems of Ecology and Evolution, Russian Academy of Sciences, Leninsky pr. 33, Moscow 119071 Russia.

${ }^{3}$ Muséum national d'Histoire naturelle, Département Ecologie et Gestion de la Biodiversité, UMR 7179 du CNRS, Equipe EVOLTRAIT, 4, Avenue du Petit Château, F-91800 Brunoy, France.
}

MOT-CLÉS: Stemmiulida, taxonomie, nouveau genre, nouvelle espèce, clé, Viet-Nam.

KEY WORDS: Stemmiulida, taxonomy, new genus, new species, key, Vietnam.

КЛЮЧЕВЫЕ СЛОВА: Stemmiulida, таксономия, новый род, новый вид, ключ, Вьетнам.

RÉSUMÉ. Description d'une nouvelle espèce (troglophile) anophthalme et dépigmentée du Viet-Nam: Eostemmiulus caecus sp.n., qui, outre qu'elle représente le premier stemmiulide rencontré dans la péninsule indochinoise, doit, par ses caractères externes (présence de filières telsoniennes, segments comprimés latéralement, structures alternées des sternites), et malgré l'absence d'yeux et de la striation primaire oblique si caractéristique du groupe, être rattachée indubitablement à l'ordre Stemmiulida. Cette nouvelle espèce, dont l'habitus rappelle celui de Stemmiulus deharvengi Mauriès \& Golovatch, 2007, d'Halmahera (Indonésie), s'en distingue nettement, comme elle se distingue des autres espèces de la même région biogéographique (Inde et Sri Lanka), mais aussi de tous les autres taxa du groupe (environ 150), répartis dans le monde entier, et donc des deux genres connus. Ce sont surtout les caractères sexuels secondaires du mâle (gonopodes, paragonopodes, premières paires de pattes), mais aussi la structure du gnathochilarium (identique à celui des Chordeumatides) et la position et la forme de la paire de filières telsoniennes qui, de par leur originalité, font les différences. C'est pourquoi nous créons ici pour elle un nouveau genre: Eostemmiulus gen.n. Ce nouveau nom suggère qu'il s'agit là pour les auteurs d'une forme dont les structures sexuelles, gonopodes et paragonopodes notamment, placent ce genre à la base d'un hypothétique arbre phylogénétique des Stemmiulida.
ABSTRACT. A new, anophthalmous and unpigmented troglophile, Eostemmiulus caecus sp.n., is described from Vietnam. Despite lacking the usual ocelli and primary oblique striations, it clearly belongs to the Stemmiulida, as based on other somatic characters (presence of spinnerets on the telson, laterally compressed segments, and alternating sternal structures) and thus represents the first record of this order from Indochina. In its habitus, the new species resembles Stemmiulus deharvengi Mauriès \& Golovatch, 2007, described from Halmahera, Indonesia, but it clearly differs from that species and all others (about 150 taxa worldwide) in the male sexual characters (gonopods, paragonopods, first pairs of legs), as well as by the structure of the gnathochilarium (identical to that of the Chordeumatida) and the surprising position and shape of the spinnerets on the telson. A new genus, Eostemmiulus gen.n., is therefore proposed to accommodate the new species. As its name suggests, the authors consider that this genus occupies a position close to the base of the phylogeny of Stemmiulida, based on male sexual characters, particularly the conformation of the gonopods and paragonopods.

РЕЗЮМЕ. Из Вьетнама описан новый слепой бесцветный трогофил: Eostemmiulus caecus sp.n. Несмотря на то, что у него нет ни глаз, ни первичной исчерченности сегментов тела, этот вид, не- 
сомненно, относится к Stemmiulida на основании других соматических признаков (наличие прядильных грифельков на тельсоне, сдавленных сбоку сегментов и сменяющихся стернальных структур), таким образом представляя первую регистрацию этого отряда в Индокитае. По своему облику новый вид походит на Stemmiulus deharvengi Mauriès \& Golovatch, 2007, описанного с острова Хальмахера (Индонезия), но отчетливо отличается от того вида и всех прочих (около 150 таксонов во всем мире) половыми признаками самца (гоноподы, парагоноподы, передние пары ног), а также структурой гнатохилярия (идентичен таковому Chordeumatida) и необычным расположением и формой прядильных грифельков на тельсоне. Поэтому для данного нового вида предложен новый род: Eostemmiulus gen.n. Как предполагает это имя, по мнению авторов, судя по половым признакам самца, прежде всего, строению гоноподов и парагоноподов, род этот занимает положение, близкое к основанию филогенетического древа Stemmiulida.

\section{Introduction}

Encore une fois, les récoltes de Louis Deharveng et Anne Bedos effectuées en région Indo-pacifique s'avèrent d'un très grand intérêt. Après la découverte, dans une cavité indonésienne de Stemmiulus deharvengi Mauriès \& Golovatch, 2007, décrit par Mauriès \& Golovatch [2007], ils nous présentent maintenant un nouveau Stemmiulida, également trouvé dans une grotte, cette fois du sud du Viet-Nam [Deharveng et al., 2009], et qui présente toute une série de caractères, sexuels ou non, qui le distinguent de tous les autres stemmiulides connus. Il présente quelques ressemblances, par son habitus, sa dépigmentation, sa petite taille, avec $S$. deharvengi, mais il est anophthalme, ce qui est rare dans ce groupe; on ne connaît en effet qu'une autre espèce aveugle, $S$. oculiscaptus Demange \& Mauriès, 1975, d'Afrique occidentale, décrite par Demange \& Mauriès [1975]. Mais c'est surtout par les caractères sexuels secondaires, observés sur les trois premières paires de pattes, les gonopodes et les paragonopodes que la nouvelle espèce se distingue, non seulement de la forme indonésienne, mais aussi des formes plus proches géographiquement (du Sri Lanka et de l'Inde) et même de toutes les formes africaines et américaines. $\mathrm{Si}$ on se souvient que toutes ces espèces ont été considérées par Mauriès \& Golovatch [2007] comme appartenant à seulement deux genres, dont un, Scoliogmus Loomis, 1941, est monospécifique, et ne diffèrent de Stemmiulus Gervais, 1844 que par des caractères externes, il est nécessaire de distinguer la nouvelle espèce de ces deux genres. C'est pourquoi nous créons ici, le nouveau genre Eostemmiulus gen.n., que nous caractérisons dans la clé qui suit. Ce nouveau genre très remarquable confirme la richesse exceptionnelle en endémiques de ces minuscules collines karstiques, extrêmement ménacées par l'exploitation industrielle de leur calcaire [Deharveng et al., 2009].

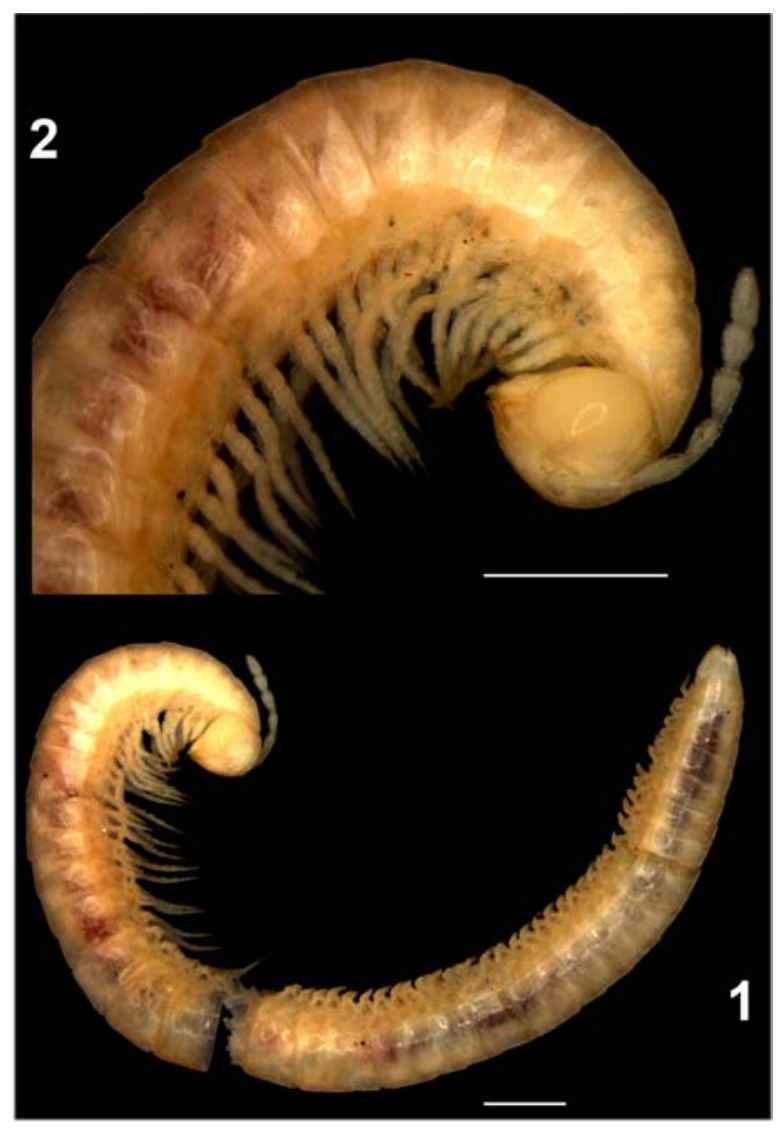

Figs 1 \& 2. Eostemmiulus caecus sp.n., o holotype: 1 Habitus photographié peu de temps après sa capture; 2 - partie antérieure du même. Echelles: 1,0 mm. Photos Louis Deharveng.

Figs 1 \& 2. Eostemmiulus caecus sp.n., $\sigma^{7}$ holotype: 1 habitus photographed soon after capture; 2 - anterior part of body of the same. Scale bars: $1.0 \mathrm{~mm}$. Photos Louis Deharveng.

Рис. 1 и 2. Eostemmiulus caecus sp.n., голотип $\sigma^{7}: 1-$ габитус, сфотографировано вскоре после поимки; 2 - передняя часть тела его же. Масштаб: 1,0 мм. Фотографии Louis Deharveng.

\section{Clé des genres de STemmiulida:}

1. Face caudale des gonopodes (P.8) à flagelles implantés parasagittalement et à mi-hauteur de l'organe. P.2 peu modifiées, à hanche normale et tractus génital constitué de deux courts manchons péniens. P.9 (paragonopodes) à télopodites tri-articulés portés par un grand sternite ..

Eostemmiulus gen.n.

- Sur la face caudale des gonopodes, les flagelles (le plus souvent pseudo-flagelles) sont implantés sur les côtés et à la base de l'organe. P.2 à hanche énorme supportant un télopodite réduit, en simple crochet ouvert; tractus génital en pénis impair. P.9 (paragonopodes) en moignons rarement articulés, portés par un sternite très réduit .. 2

2. Stipes du gnathochilarium du $\sigma^{7}$ pourvus d'un important lobe latéral; stries métazonitales les plus dorsales fortement courbées .................... Scoliogmus Loomis, 1941

- Stipes du gnathochilarium du $\sigma^{7}$ sans lobe latéral; stries métazonitales les plus dorsales droites, rarement effacées Stemmiulus Gervais, 1844 


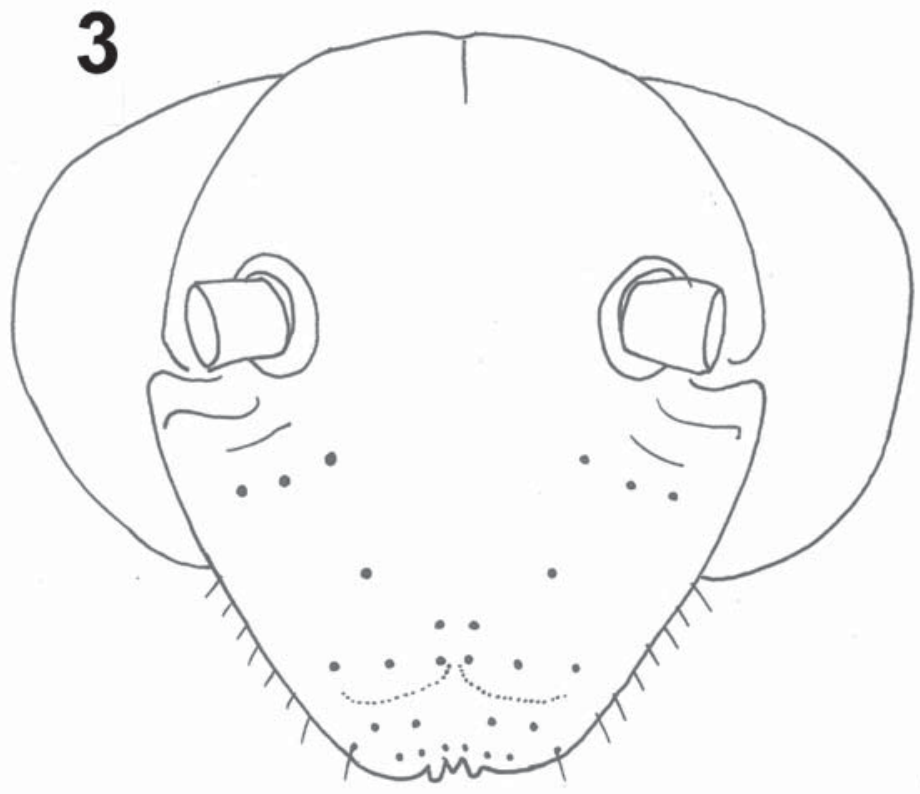

6
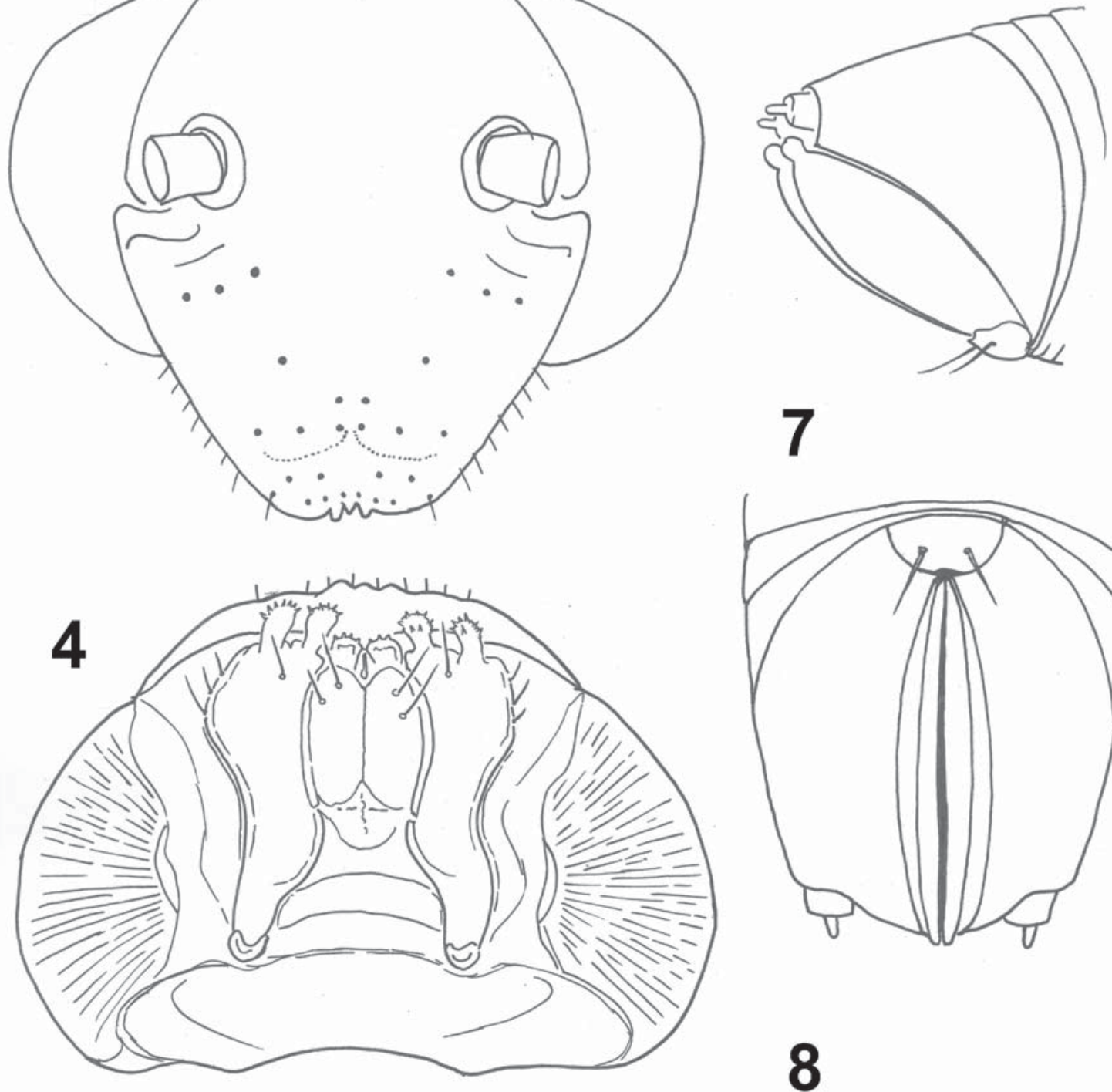

7
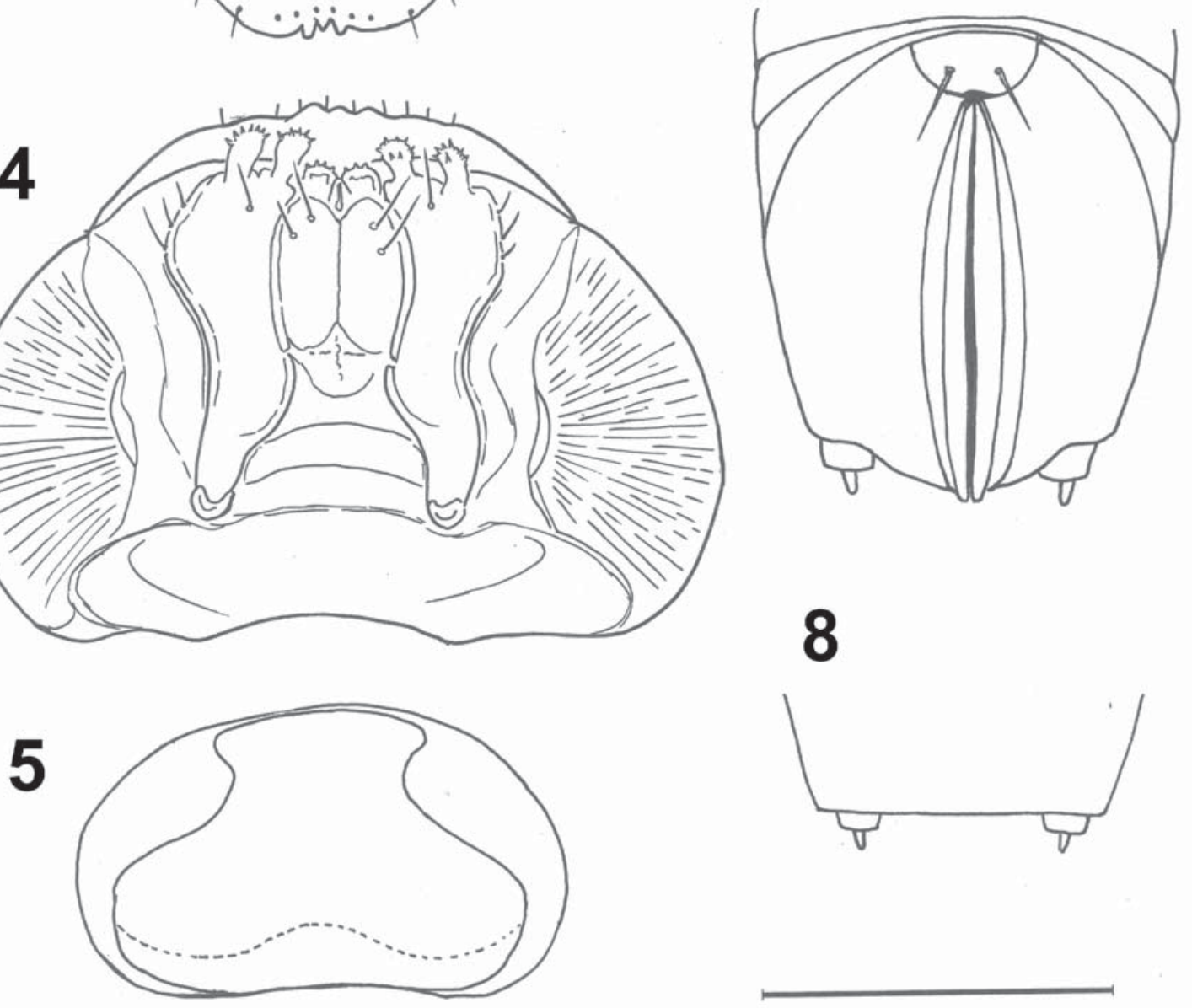

Figs 3-8. Eostemmiulus caecus sp.n., O' holotype: 3 - tête, vue de face; 4 - la même, face ventrale; 5 - collum, face ventrale; 6 telson, vue latérale droite; 7 - le même, face ventrale; 8 - extrémité du même, vue dorsale. Échelle $=0,5 \mathrm{~mm}$.

Figs 3-8. Eostemmiulus caecus sp.n., $\sigma^{7}$ holotype: 3 - head, front view; 4 - same, ventral view; 5 - collum, ventral view; 6 telson, right lateral view; 7 - same, ventral view; 8 - caudal part of the same, dorsal view. Scale bar: $0.5 \mathrm{~mm}$.

Рис. 3-8. Eostemmiulus caecus sp.n., голотип О': 3 - голова, вид спереди; 4 - то же, вид снизу; 5 - коллум, вид снизу; 6 тельсон, вид справа и сбоку; 7 - то же, вид снизу; 8 - каудальная часть его же, вид сверху. Масштаб: 0,5 мм. 


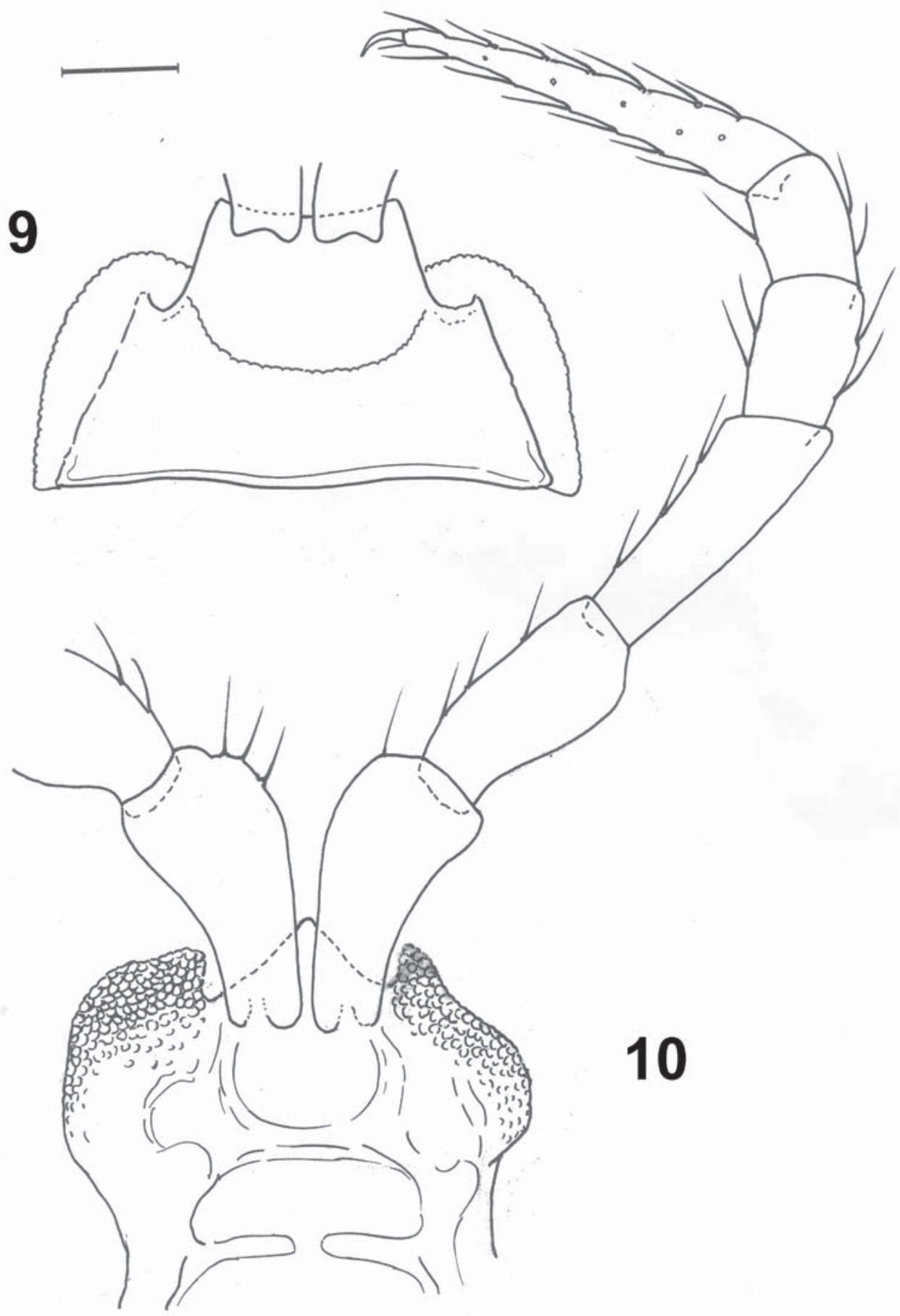

Figs 9 \& 10. Eostemmiulus caecus sp.n., 3 holotype: 9 - sternite isolé de la P.6; 10 - P.7, face caudale. Échelle $=0,1$ mm.

Figs $9 \&$ 10. Eostemmiulus caecus sp.n., $0^{7}$ holotype: 9 - isolated sternite of leg-pair 6; 10 - leg-pair 7, caudal view. Scale bar: 0.1 $\mathrm{mm}$.

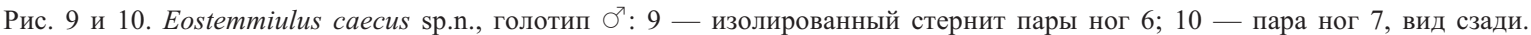
Масштаб: $0.1 \mathrm{мм.}$ 


\section{Eostemmiulus gen.n.}

DIAGNOSE $\sigma^{7}:$ P.2 peu déformées, à tractus génital en deux moignons péniens. Face caudale des gonopodes à flagelles implantés parasagittalement et dans la partie distale des hanches. Paragonopodes à sternite bien développé supportant une paire de télopodites tri-articulés. Gnathochilarium du type Chordeumatida. Deux filières telsoniennes nettement écartées l'une de l'autre.

Espèce-type: Eostemmiulus caecus sp.n.

\section{Eostemmiulus caecus sp.n.}

Figs 1-17.

HOLOTYPE $\sigma^{7}$ (MNHN Collection Myriapoda-Onychophora DC 041), Viet-Nam, Kien Giang Province, Kien Luong, Hon Chong, Nui Hills, Bai Voi, hospital cave Mo So, by hand, 19/12/1994, Deharveng, Dung \& Le Cong Kiet leg. (VIET-046).

CARACTERES MORPHOLOGIQUES EXTER-

NES. Corps entièrement blanc, dépigmenté, après long séjour en alcool, paraissant teinté de brun clair sur les photos prises peu après la capture par les collecteurs (Figs $1 \& 2$ ).

Longueur du corps $16 \mathrm{~mm}$; segments moyens: 1,16 $\mathrm{mm}$ de diamètre vertical, $0,88 \mathrm{~mm}$ de diamètre horizontal.

41 anneaux (telson inclus); 69 paires de pattes (gonopodes et paragonopodes comptablisés comme pattes ambulatoires); 3 anneaux prételsoniens apodes.

Tête (Figs 3 \& 4) à capsule céphalique à suture occipitale, mais sans sutures latérales, à faible pilosité faciale; yeux absents (comme chez S. oculiscaptus de Côte d'Ivoire); labre tridenté; joues normalement saillantes; gnathochilarium à mentum et promentum membraneux transparents, et se distinguant surtout des autres stemmiulides par la forme des stipes qui au lieu d'avoir leurs bords latéraux subparallèles, s'élargissent ici de la base au sommet comme dans l'ordre voisin Chordeumatida.

Les antennes, perdues, sont visibles seulement sur les photos (Figs 1 \& 2); sans particularité notable, elles mesurent $1,5 \mathrm{~mm}$ de long, et la massue antennaire (antennomères claviformes V à VIII) est environ 5 fois plus longue que large.

Collum (Fig. 5) sans aucune ornementation.

Segments moyens: presque entièrement lisses surtout dorsalement, dépourvus de la striation oblique typique des stemmiulides. Sur les flancs des métazonites, en arrière d'une ligne de suture très fine mais bien visible, on peut distinguer des strioles longitudinales très faiblement marquées, très serrées, souvent interrompues et plus ou moins ondulées.

Telson (Figs 6-8) remarquable par la position très latérale de chaque élément de la paire de filières, qui sont parasagittales chez tous les stemmiulides. Ces filières, qui sont en général chez les stemmiulides déjà connus de simples boutons ou de courtes digitations, présentent ici une structure très particulière. Leur partie distale, simple courte digitation, est portée par une robuste base cylindrique qui recèle peut-être une glande séricigène.
CARACTÈRES SEXUELS SECONDAIRES ${ }^{7}$ :

Les premières paires de pattes ne présentent aucune des transformations que l'on observe chez les autres stemmiulides:

Les P.1 (Fig. 11) sont courtes, mais sans particularités notables.

Les P.2 (Fig. 12) sont très semblables aux P.1 par leurs télopodites, et ne ressemblent en rien aux P.2 très modifiés des autres stemmiulides; elles présentent un tractus génital constitué d'une paire de manchons péniens, au lieu de l'habituel pénis impair!

Les P.3 (Fig. 13) ont la particularité d'avoir des hanches munies chacune d'un long prolongement digitiforme érigé.

Les P.4 à P.7, comme d'ailleurs toutes les pattes post-gonopodiales, ont des télopodites relativement grêles, comme celui de la figure 10. Les figures 9 (P.6) et 10 (P.7) montrent les sternites tels qu'ils apparaissent sur toutes les pattes paires (comme sur les P.6) ou impaires (comme sur les P.7), alternance caractéristique des stemmiulides.

Les gonopodes (Figs 15-17) n'entrent dans aucune des catégories définies par Demange \& Mauriès [1975]; ils se distinguent de tous les gonopodes connus chez les stemmiulides par un fort développement de la zone sterno-trachéenne (S), qui est pratiquement inexistante chez tous les autres représentants de l'ordre. Cette zone basale est surmontée par une zone bien développée et musculeuse constituée d'une paire de hanches en trapèze nettement plus longs que larges (angiocoxites, $\mathrm{K}$ ); chaque hanche se prolonge par une longue et étroite bande parasagittale érigée à sommet arrondi (colpocoxite, k), aplatie en ruban. A la base de chaque prolongement parasagittal, sur la face caudale, naît un vrai flagelle (la plupart des flagelles de stemmiulides sont des pseudo-flagelles) à base renflée semblable à celui des gonopodes de Julida ou de Chordeumatida, et qui ne semble pas être engainé dans quelque structure engainante. La position de ces flagelles est complètement différente de celle des autres stemmiulides, puisqu'ils s'implantent ici non à la base et sur les côtés du gonopode, mais en position parasagittale et à mi-hauteur de ce dernier. Dans la partie distale du gonopode, à l'extérieur du prolongement $(\mathrm{k})$, se trouve un télopodite en crochet ouvert $(\mathrm{T})$. De même longueur que $(\mathrm{k})$, ce télopodite s'articule à la hanche, comme le montre la figure 17 où on peut voir l'apodème (caché par une petite expansion en lame angulaire chitineuse latérodistale $(\mathrm{m})$ ) où s'attache une puissante musculature. Il n'existe pas de télopodite autonome, et a fortiori de télopodite articulé à la hanche, chez les autres Stemmiulida.

Chaque moitié de la partie distale du gonopode (Fig. 17) porte 9 fortes soies: une très longue sur la face caudale du prolongement $(\mathrm{k})$, une à l'apex de la lame $(\mathrm{m})$, cinq sur le télopodite (trois sur la face orale et deux sur la face caudale), et enfin deux dans le rebord chitineux qui relie $(\mathrm{k})$ à la base du télopodite (T). 

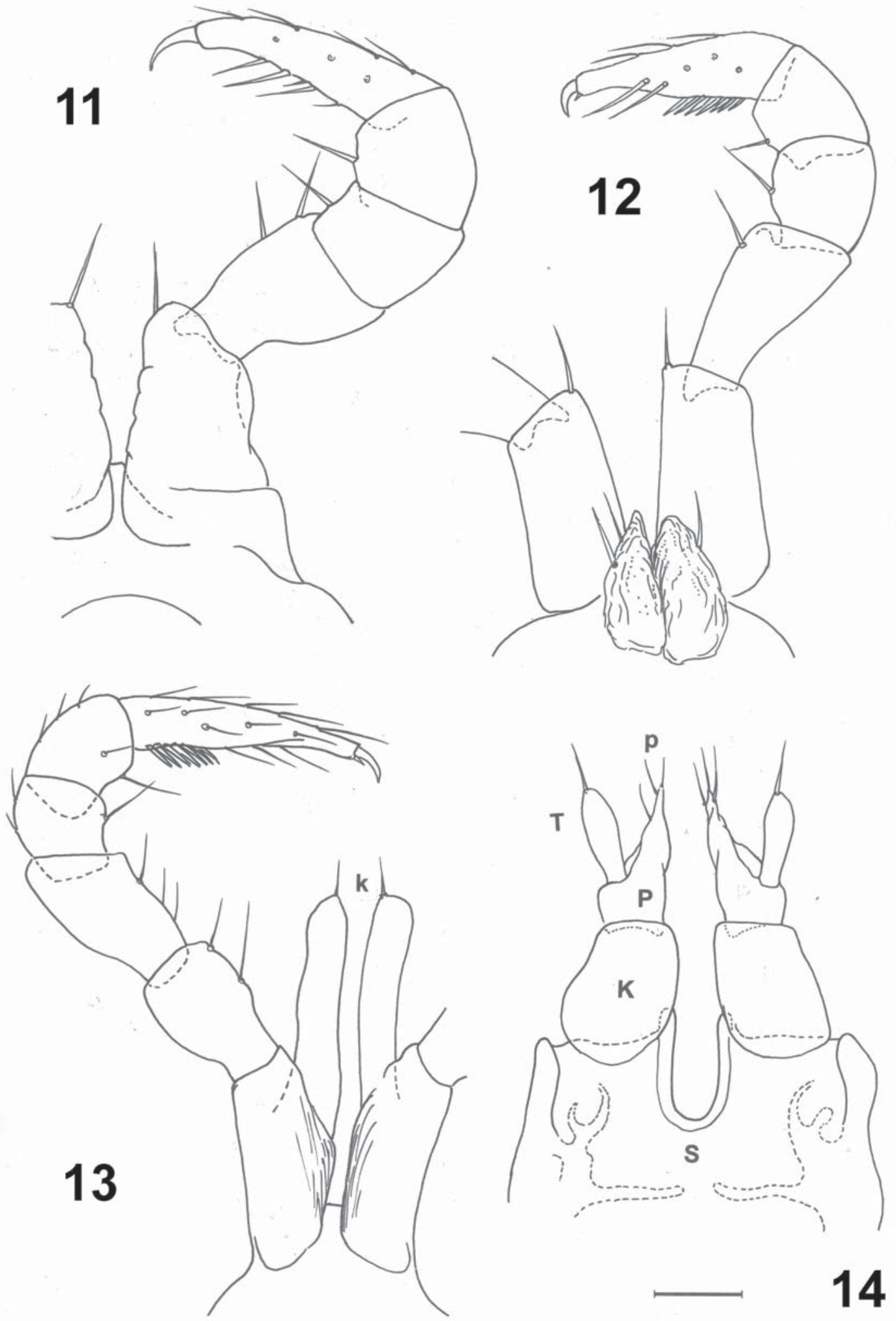

Figs 11-14. Eostemmiulus caecus sp.n., O' holotype: $11-$ P.1, face caudale; $12-$ P. 2 et bourgeons péniens, face caudale; $13-$ P.3, face caudale; $14-$ P.9 (paragonopodes), face caudale. Échelle $=0,1 \mathrm{~mm}$.

Figs 11-14. Eostemmiulus caecus sp.n., $0^{7}$ holotype: 11 - leg-pair 1, caudal view; 12 - leg-pair 2 and penial sacs, caudal view; 13 - leg-pair 3, caudal view; 14 - leg-pair 9 (paragonopods), caudal view. Scale bar: $0.1 \mathrm{~mm}$.

Рис. 11-14. Eostemmiulus caecus sp.n., голотип О7: 11 - пара ног 1, вид сзади; 12 - пара ног 2 и мешочки пениса, вид сзади; 13 - пара ног 3, вид сзади; 14 - пара ног 9 (парагоноподы), вид сзади. Масштаб: 0,1 мм. 


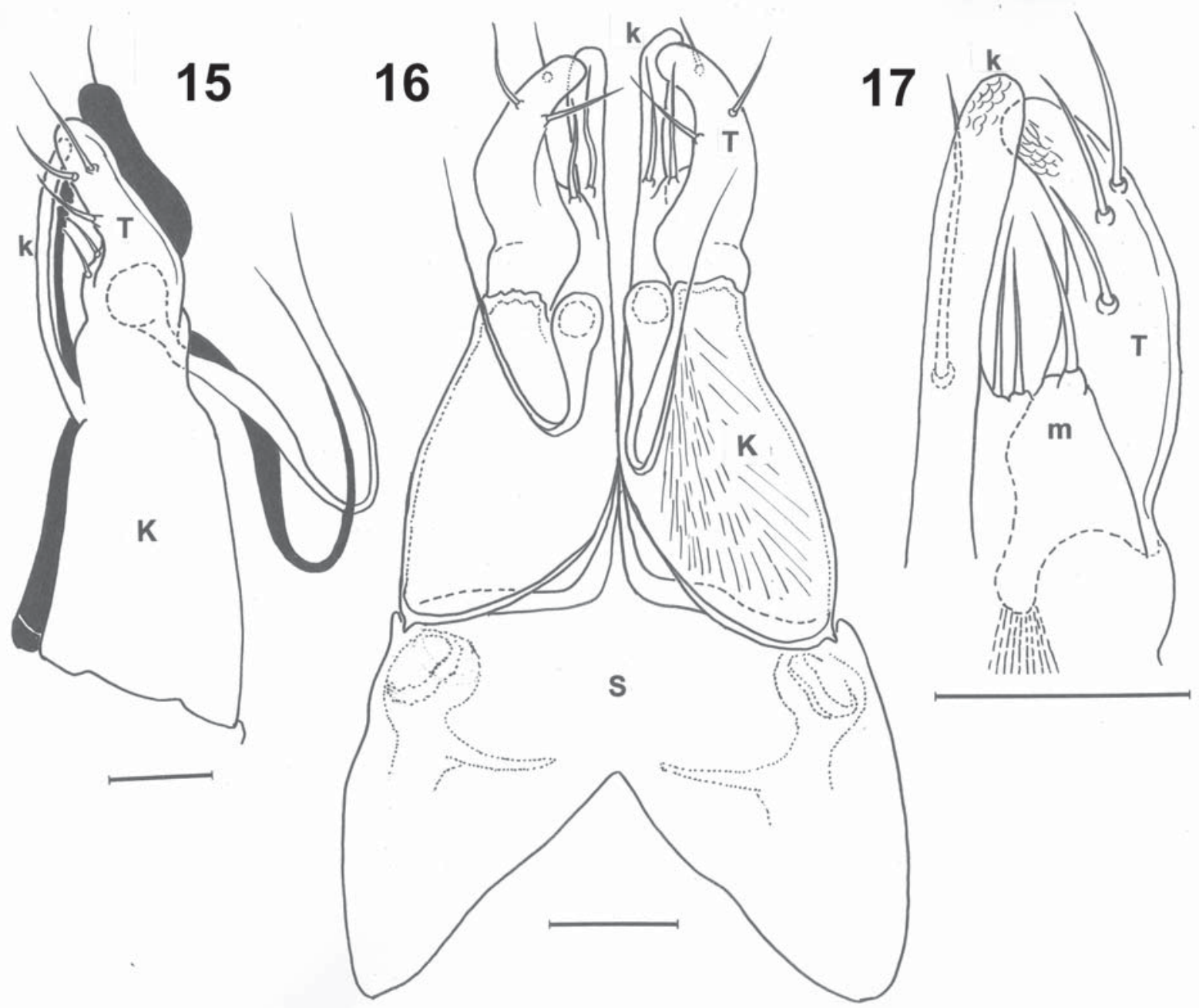

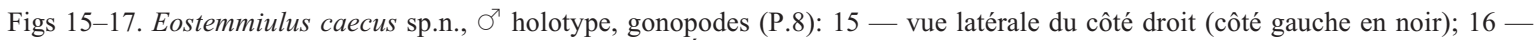
face caudale; 17 - face orale de la partie distale du côté droit. Échelles $=0,1 \mathrm{~mm}$.

Figs 15-17. Eostemmiulus caecus sp.n., $\sigma^{\top}$ holotype, gonopods (leg-pair 8): 15 - lateral view from right side (left side in black); 16 - caudal view; 17 - oral view of the distal part from right side. Scale bars: $0.1 \mathrm{~mm}$.

Рис. 15-17. Eostemmiulus caecus sp.n., голотип О', гоноподы (пара ног 8): 15 - вид сбоку с правой стороны (левая сторона зачернена); 16 - вид сзади; 17 - вид спереди дистальной части с правой стороны. Масштаб: 0,1 мм.

Paragonopodes (Fig. 14) se distinguant de tous les paragonopodes de stemmiulides par la présence d'un sternite bien développé, en large bandeau profondément échancré médialement et qui porte, non une paire de moignons, mais une paire de membres triarticulés. Chacun de ces membres comprend une hanche $(\mathrm{K})$ subcylindrique surmontée d'un préfémur $(\mathrm{P})$ qui porte le reste du télopodite $(\mathrm{T})$ constitué d'un seul article claviforme muni d'une soie apicale. Le préfémur $(\mathrm{P})$ émet un prolongement interne aigu (p) porteur de trois soies apicales.

Femelle inconnue.

\section{Conclusion}

Mauriès \& Golovatch [2007] soulignaient la grande uniformité de l'ordre pantropical Stemmiulida, dont les ca 150 espèces se répartissaient selon eux en deux genres seulement, dont l'un, Scoliogmus Loomis, 1941, est monospécifique! Toutes les autres espèces étaient réunies dans le grand genre Stemmiulus. Cette remarquable stabilité et uniformité est très sérieusement mise en brèche par la découverte de Louis Deharveng, qui nous révèle l'existence d'une forme présentant toute une série de caractères sensiblement différents de ceux 
que l'on rencontre chez tous les autres Stemmiulida. Il ne fait pas de doute pour nous que, malgré l'existence de traits hautement significatifs de la troglomorphie (absence d'yeux, dépigmentation), les caractères sexuels notamment traduisent une primitivité certaine qui place le genre Eostemmiulus gen.n. à la base d'un hypothétique arbre phylogénétique du groupe. Certains caractères (gnathochilarium, vrais flagelles des gonopodes) semblent corroborer ce que l'un de nous suggérait [Mauriès, 1978]: les Stemmiulida pourraient être considérés comme groupe frère de l'ordre Chordeumatida.

REMERCIEMENTS. Le second auteur exprime sa reconnaissance au Muséum national d'Histoire naturelle (Paris, France) pour le support financier qui a rendu possible son séjour en mars 2010, et aussi au personnel de la section Zoologie-Arthropodes du Département Systématique \& Evolution, et notammment à Christine Rollard, responsable des Arthropodes terrestres. Grand merci aussi à Louis Deharveng, Le Cong Kiet et Duong Tien Dung, inventeurs du matériel si exceptionellement intéressant qu'ils ont eu l'amabilité de nous confier, ainsi qu'à Louis Deharveng pour les photos qu'il nous a autorisé à utiliser pour cette publica- tion. Nos remerciements s'adressent également à Natdainai Likhitrakarn (Université Chulalongkorn, Bangkok, Thaïlande) pour son assistance technique.

\section{Références}

Deharveng L., Bedos A., Le Cong Kiet, Le Cong Man, Truong Quang Tam. 2009. Endemic arthropods of the Hon Chong hills (Kien Giang), an unrivaled biodiversity heritage in Southeast Asia // Le Cong Kiet, Truong Quang Tam, Ly Ngoc Sam (eds.). Beleagered Hills: Managing the biodiversity of the remaining karst hills of Kien Giang, Vietnam. Ho Chi Minh: Nha Xuat Ban Nong Nghiep, TP. P.31-57.

Demange J.-M., Mauriès J.-P. 1975. Myriapodes-Diplopodes des Monts Nimba et Tonkoui (Côte d'Ivoire, Guinée) récoltés par M. Lamotte et ses collaborateurs de 1942 à 1960 // Ann. Mus. Royal Afr. Centr., sér. in- ${ }^{\circ}$, Sci. Zool. No.212. P.1-192.

Loomis H.F. 1941. Millipeds collected in Puerto-Rico and the Dominican Republic by Dr. P.J. Darlington in 1938 // Bull. Mus. Comp. Zool., Harv. Univ. Vol.88. No.2. P.17-80.

Mauriès J.-P. 1978. Le genre néo-zélandais Schedotrigona Silvestri, 1903: révision et place dans une nouvelle classification des Craspédosomides (Myriapoda, Diplopoda, Craspedosomida) // Bull. Mus. natn. Hist. nat., Paris. $3^{\text {ème }}$ sér. No.510, Zool.351. P.43-66.

Mauriès J.-P., Golovatch S.I. 2007. Stemmiulus deharvengi sp.n., le premier Stemmiulida signalé en Indonésie (Diplopoda: Stemmiulida) // Arthropoda Selecta. Vol.15. No.2. P.91-98 